\title{
A hierarchy of modal logics with relative accessibility relations
}

\author{
Philippe Balbiani and Ewa OrŁowska \\ Laboratoire d'informatique de Paris-Nord* \\ Institute of Telecommunications, Warsaw ${ }^{\dagger}$
}

\begin{abstract}
In this paper we introduce and investigate various classes of multimodal logics based on frames with relative accessibility relations. We discuss their applicability to representation and analysis of incomplete information. We provide axiom systems for these logics and we prove their completeness.
\end{abstract}

\section{Introduction}

The original motivation for introducing and investigating modal logics with relative accessibility relations comes from the theory of information systems. However, as it is shown in this paper, a number of standard multimodal logics can be uniformly presented and investigated within the general framework of relative relations as well. Information systems are the collections of information items that have the form of descriptions of some objects in terms of their properties. More formally, by an information system we mean a structure $S=\left(O B, A T,\left\{V A L_{a}: a \in A T\right\}\right)$ such that $O B$ is a nonempty set

*Philippe Balbiani, balbiani@lipn.univ-paris13.fr, Laboratoire d'informatique de ParisNord, Avenue Jean-Baptiste Clément, 93430 Villetaneuse.

${ }^{\dagger}$ Ewa Orlowska, orlowska@itl.waw.pl, Institute of Telecommunications, ul. Szachowa 1, 04-894 Warsaw. 
of objects, $A T$ is a finite nonempty set of attributes, each $V A L_{a}$ is a nonempty set of values of attribute $a$. Each attribute is a function $a$ : $O B \rightarrow \mathcal{P}\left(V A L_{a}\right)$ that assigns subsets of values to objects. Any set $a(x)$ can be viewed as a set of properties of an object $x$.

For example, if the attribute $a$ is "colour" and $a(x)=\{$ green $\}$, then $x$ possesses the property of "being green"; if $a$ is "age" and $x$ is 25 years old, then $a(x)=\{25\}$ and this means that $x$ possesses the property of "being 25 years old" ; if $a$ is "languages spoken" and if a person $x$ speaks, say, Polish $(P l)$, German $(D)$ and French $(F)$, then $a(x)=\{P l, D, F\}$ and $x$ possesses the properties of "speaking Polish", "speaking German" and "speaking French". In this setting any set $a(x)$ is referred to as the set of $a$-properties of object $x$ and its complement $V A L_{a} \backslash a(x)$ is the set of negative $a$-properties of $x$. Consider, for example, the file given below :

$$
\left.\begin{array}{c|c} 
& \text { colour } \\
o_{1} & \text { green } \\
o_{2} & \text { green } \\
o_{3} & \text { blue } \\
o_{4} & \text { blue } \\
o_{5} & \text { red }
\end{array}\right)
$$

In that file, we have $O B=\left\{o_{1}, o_{2}, o_{3}, o_{4}, o_{5}\right\}$ and $A T=\{$ colour $\}$. Moreover, $V A L_{\text {colour }}=\{$ green, blue, red $\}$. Suppose that we are interested in defining a set $X=\left\{o_{2}, o_{3}, o_{4}\right\}$ in terms of information provided in the file. The set might be identified with a concept, say, the concept "beautiful objects". We easily observe that the following statement is not true of the given objects : $o \in X$ iff $o$ is either green or blue. The reason being that the information that is available in the file does not enable us to discern between $o_{1}$ and $o_{2}$. We observe that the attribute "colour" induces a relation in the set of objects (referred to as an indiscernibility relation) that reflects their indistinguishability in terms of colour :

$$
\text { - }\left(o, o^{\prime}\right) \in \text { ind (colour) iff colour }(o)=\operatorname{colour}\left(o^{\prime}\right) \text {. }
$$

This suggests that, given an information system $S=\left(O B, A T,\left\{V A L_{a}\right.\right.$ : $a \in A T\}$ ), the properties of objects available in $S$ induce relationships among the objects. Typically, these relationships have the form of binary relations. They are referred to as information relations. There are two major groups of information relations : the relations 
that reflect various forms of indistinguishability of objects in terms of their properties and the relations that express distinguishability of the objects. In the following we present few examples of information relations. Let $A$ be a subset of the set $A T$ of attributes. The most familiar family of information relations that can be derived from $S$ is the family of indiscernibility relations:

- strong indiscernibility : $\left(o, o^{\prime}\right) \in \operatorname{ind}(A)$ iff $a(o)=a\left(o^{\prime}\right)$ for all $a \in A$.

These relations are strong indiscernibility relations, they hold between two objects whenever the objects are "the same" with respect to their $a$-properties for all $a \in A$. Clearly, it is also reasonable to consider weak indiscernibility, that is indistinguishability of objects with respect to some, not necessarily all the properties :

- weak indiscernibility $:\left(o, o^{\prime}\right) \in$ wind $(A)$ iff $a(o)=a\left(o^{\prime}\right)$ for some $a \in A$.

Another family of useful information relations is the family of similarity relations:

- strong (weak) similarity : $(x, y) \in \operatorname{sim}(A)(w \operatorname{sim}(A))$ iff $a(x) \cap$ $a(y) \neq \emptyset$ for all (some) $a \in A$.

Indiscernibility and similarity relations exhibit indistinguishability of objects. We might also be interested in deriving information about distinguishability. The following are examples of the families of information relations that reflect differences between the objects:

- strong (weak) diversity : $(x, y) \in \operatorname{div}(A)(w \operatorname{div}(A))$ iff $a(x) \neq$ $a(y)$ for all (some) $a \in A$,

- strong (weak) orthogonality : $(x, y) \in \operatorname{ort}(A)(\operatorname{wort}(A))$ iff $a(x) \cap$ $a(y)=\emptyset$ for all (some) $a \in A$,

- strong (weak) complementarity : $(x, y) \in \operatorname{com}(A)(w \operatorname{com}(A))$ iff $a(y)$ is the complement of $a(x)$ with respect to the set $V A L_{a}$, for all (some) $a \in A$.

An information system constitutes an explicit information available in an application domain, while information relations are an implicit information. These relations enable us to identify some aspects of incompleteness of explicit information.

Relational systems consisting of a family of relations on a set are 
referred to as frames. By a frame derived from an information system $S=\left(O B, A T,\left\{V A L_{a}: a \in A T\right\}\right)$ we mean a relational system $K_{S, R}=(O B,\{R(A): A \subseteq A T\})$, where $\{R(A): A \subseteq A T\}$ is any of the families of information relations. Observe that relations in these frames depend on the subsets of the set $A T$. In a general setting these subsets play the role of parameters which provide a means for representing an intensional part of information included in an information system. From a technical point of view, we deal with families of relations indexed with subsets of a set.

Frames with relative accessibility relations have been suggested in Orlowska [20] in the context of a rough set analysis of data, and they were investigated, among others, in Konikowska [15] [16] and Balbiani [2] [3]. Often we are also interested in studying relationships between information relations that belong to different families. Hence, it is also natural to consider frames with families of relations of different types. A great variety of such frames is studied in the literature, see for example Demri [5] [6], Demri and Orłowska [7], Orłowska [18] [19] [21] [23] and Vakarelov [25] [26] [27].

The motivation for using modal logics for reasoning about information relations comes from the methods of data analysis in information systems. In these methods, the modal-like operators are used in the languages for representation of incomplete information. Let $S=\left(O B, A T,\left\{V A L_{a}: a \in A T\right\}\right)$ be an information system. Given a strong indiscernibility relation $\operatorname{ind}(A)$, for $A$ being a subset of $A T$, and a subset $X$ of $O B$, the lower $A$-approximation of $X$ and the upper $A$-approximation of $X$ are defined as follows :

- $L(A) X=\{x \in O B$ : for all $y \in O B$, if $(x, y) \in \operatorname{ind}(A)$ then $y \in X\}$.

- $U(A) X=\{x \in O B$ : there is $y \in O B$ such that $(x, y) \in \operatorname{ind}(A)$ and $y \in X\}$.

The following hierarchy of definability of sets is obtained in a natural way in terms of the approximations. A subset $X$ of $O B$ is said to be :

- A-definable if $L(A) X=X$ (or equivalently $U(A) X=X$ ).

- Roughly $A$-definable if $L(A) X \neq \emptyset$ and $U(A) X \neq O B$.

- Internally $A$-indefinable if $L(A) X=\emptyset$.

- Externally $A$-indefinable if $U(A) X=O B$. 
- Totally $A$-indefinable if internally $A$-indefinable and externally $A$-indefinable.

$A$-definability of a set $X$ means that $X$ is the union of some of the equivalence classes of $i n d(A)$. In our example $L$ (colour) $X=\left\{o_{3}, o_{4}\right\}$ and $U($ colour $) X=\left\{o_{1}, o_{2}, o_{3}, o_{4}\right\}$ and we conclude that $X$ is not definable in terms of colour. Clearly, $X$ cannot be covered with the equivalence classes $\left\{o_{1}, o_{2}\right\},\left\{o_{3}, o_{4}\right\},\left\{o_{5}\right\}$ of ind(colour). From the perspective of concept analysis any subset of objects in an information system might be identified with an extension of a concept and any subset of attributes with an intension of a concept.

Following the rough set semantics of vague concepts developed in Orlowska [17] and Read [24], we define the sets of $A$-positive, $A$ borderline and $A$-negative instances of a set $X$ of objects as follows :

- $\operatorname{POS}(A) X=L(A) X$.

- $B O R(A) X=U(A) X \backslash L(A) X$.

- $N E G(A) X=O B \backslash U(A) X$.

Elements of $P O S(A) X$ definitely, relative to properties corresponding to $A$, belong to $X$. Elements of $N E G(A) X$ definitely, up to these properties, do not belong to $X$. BOR $(A) X$ is a doubtful region, its elements possibly belong to $X$, but we cannot decide it for certain considering only properties corresponding to $A$. In other words, as far as indiscernibility $\operatorname{ind}(A)$ is concerned, nothing can be said about membership to $X$ of elements from $B O R(A) X$.

The above analysis suggests that it might be useful to define the operators analogous to the lower and upper approximation also with the other information relations. These operators enable us to disclose an interaction between the information relations and subsets of objects. Let $S=\left(O B, A T,\left\{V A L_{a}: a \in A T\right\}\right)$ be an information system and let $\{R(A): A \in A T\}$ be a family of information relations derived from $S$. There are two major groups of operators :

- $[R(A)] X=\{x \in O B$ : for all $y \in O B$, if $(x, y) \in R(A)$ then $y \in X\}$.

- $\langle R(A)\rangle X=\{x \in O B$ : there is $y \in O B$ such that $(x, y) \in R(A)$ and $y \in X\}$.

Clearly, from the logic point of view they are necessity and possibility operators, respectively, and information relations play the role of the 
accessibility relations that determine these modal operators. Hence, modal logics appear to be a natural formal tool for the analysis of data. However, to represent adequately all the ingredients of information provided in an information system, we need to make the accessibility relations relative. Since the information relations derived from information systems always provide a twofold information, namely, the information which objects are related and the information with respect to which attributes these objects are related, in an abstract setting of modal logics we need the relations that are relative to subsets of a set. In this context, several modal logics have been introduced. Their linguistic basis is the propositional calculus enriched, for every expression ? in some language of parameters, with the modality [?]. The language of parameters differs from one logic to another.

In the context of the modal logics for information systems introduced by Orlowska [20], one has to consider parameter expressions? defined by the atomic parameters and the operation $\cap$. These expressions enable us to represent strong information relations. In the relational semantics of these logics, the accessibility relation $R$ is parametrized in such a way that for every parameter ?, $\Delta, R(? \cap \Delta)=R(?) \cap R(\Delta)$. In the context of $D A L$, the modal logic for data analysis introduced by Fariñas del Cerro and Orłowska [9], the parameter expressions ? are built up from atomic expressions with the operations $\cap$ and $\cup^{\star}$ corresponding to the interpretation of the compound accessibility relations defined with intersection and transitive closure of union. In the relational semantics of these logics, the accessibility relation $R$ is parametrized in such a way that for every parameter?, $\Delta, R(? \cap \Delta)=$ $R(?) \cap R(\Delta)$ and $R\left(? \cup^{\star} \Delta\right)=R(?) \cup^{\star} R(\Delta)$.

In the context of $B M L$, the Boolean modal logic introduced by Gargov, Passy and Tinchev [11] [12], the parameter expressions ? are built up from atomic expressions with the operations $\cap, \cup$ and $\neg$ corresponding to the interpretation of the compound accessibility relations defined with intersection, union and complement. In the relational semantics of these logics, the accessibility relation $R$ is parametrized in such a way that for every parameter ?, $\Delta, R(? \cap \Delta)=R(?) \cap R(\Delta)$, $R(? \cup \Delta)=R(?) \cup R(\Delta)$ and $R(\neg ?)=\overline{R(?)}$.

Several modal logics for information systems have been considered, depending on the special properties of the relative accessibility relation. If for every parameter?, $R($ ? ) is a relation of equivalence then the accessibility relation is a relation of strong indiscernibility. Such frames 
$\left(W,\{R(?)\}_{\Gamma}\right)$ correspond to the frames of indiscernibility associated to attribute systems. If for every ?, $R($ ?) is reflexive and symmetric then the accessibility relation is a relation of strong similarity and the frames $\left(W,\{R(?)\}_{\Gamma}\right)$ are the frames of similarity associated to attribute systems.

In the section 2 of this paper, we consider several properties of the relative accessibility relations $R(?)$ where the parameters are defined by the operation $\cap$ in such a way that, in the relational semantics of these logics, $R(? \cap \Delta)=R(?) \cap R(\Delta)$ and we prove the axiomatizability of the formulas valid in the corresponding class of frames. In the section 3, we consider an extended language where the parameters are defined by the operations $\cap$ and $U$ in such a way that, in the relational semantics of these logics, $R(? \cap \Delta)=R(?) \cap R(\Delta)$ and $R(? \cup \Delta)=R(?) \cup R(\Delta)$. Therefore, this extended language is a sublanguage of the Boolean modal logic [11] [12], only the parameters of the form $\neg$ ? are missing. We examine the axiomatizability of the set of formulas valid in several classes of frames and we prove the completeness of these axiomatizations. Our proof is based on the techniques of the copying introduced by Vakarelov [26].

\section{Modal logics $L 2$}

In this section we present a class $L 2$ of relative modal logics where the parameters are defined by the operation $\cap$ in such a way that, in the relational semantics of these logics, $R(? \cap \Delta)=R(?) \cap R(\Delta)$.

\subsection{Language}

The linguistic basis of any modal logic from class $L 2$ is the language of the classical propositional calculus enriched with modal operators. Each modal operator is denoted by an expression that represents a set of parameters. Let $A P A R$ be a nonempty set of atomic parameters. The set $C P A R$ of the complex parameters is defined by induction in the following way:

- Every atomic parameter is a complex parameter,

- For every ?, $\Delta \in C P A R, ? \cap \Delta \in C P A R$.

Let set be the mapping of $C P A R$ into $2_{f}^{A P A R}$ - the set of the finite subsets of $A P A R$ - defined by induction in the following way : 
- For every atomic parameter $\alpha, \operatorname{set}(\alpha)=\{\alpha\}$,

- For every ?, $\Delta \in C P A R, \operatorname{set}($ ? $\cap \Delta)=\operatorname{set}($ ? $) \cup \operatorname{set}(\Delta)$.

In what follows, $X \subseteq_{f} Y$ means that $X$ is a finite subset of $Y$. Observe that :

Theorem 1 For every $P \subseteq_{f} A P A R$, there is $\in C P A R$ such that $\operatorname{set}(?)=P$.

Let $\sqsubseteq$ be the binary relation on $C P A R$ defined in the following way :

- For every ?, $\Delta \in C P A R, ? \sqsubseteq \Delta$ iff $\operatorname{set}(?) \subseteq \operatorname{set}(\Delta)$.

If the expressions ? and $\Delta$ are considered as Boolean formulas of the classical propositional calculus, then one can easily prove that:

- ? $\sqsubseteq \Delta$ iff $\Delta \rightarrow$ ? is classically valid.

Let $A F O R$ be a nonempty set of atomic formulas. The set $C F O R$ of the complex formulas is defined by induction in the following way:

- Every atomic formula is a complex formula,

- For every $A \in C F O R, \neg A \in C F O R$,

- For every $A, B \in C F O R, A \wedge B \in C F O R$,

- For every ? $\in C P A R$ and for every $A \in C F O R,[?] A \in C F O R$.

For every ? $\in C P A R$ and for every $A \in C F O R$, let $\langle ?\rangle A=\neg[?] \neg A$.

\subsection{Semantical study}

A frame for $L 2$ is a relational structure of the form $\mathcal{F}=(P A R, O B, R)$ where:

- $P A R$ is a nonempty set of parameters,

- $O B$ is a nonempty set of objects,

- $R$ is a mapping of $2_{f}^{P A R}$ - the set of the finite subsets of $P A R$ - into the set of the binary relations on $O B$ such that, for every $P, Q \subseteq_{f} P A R, R(P \cup Q) \subseteq R(P) \cap R(Q)$.

Throughout section 2, by "frame" we always mean a frame for $L 2$. $\mathcal{F}$ is standard when, for every $P, Q \subseteq_{f} P A R, R(P \cup Q)=R(P) \cap R(Q)$. Let $m, n, j, k \geq 0 . \mathcal{F}$ is ${ }_{j k}^{m n}$-normal when, for every $P \subseteq_{f} P A R$ and for every $x, y, z \in O B$, if $x R(P)^{m} y$ and $x R(P)^{j} z$ then there is $t \in O B$ such that $y R(P)^{n} t$ and $z R(P)^{k} t$ where: 
- $R(P)^{0}=I d_{O B}$

- $R(P)^{s+1}=R(P)^{s} ; R(P)$, where ; is the composition of relations.

For example :

- $\mathcal{F}$ is ${ }_{00}^{01}$-normal when each $R(P)$ is reflexive.

- $\mathcal{F}$ is ${ }_{00}^{11}$-normal when each $R(P)$ is symmetric.

- $\mathcal{F}$ is ${ }_{20}^{01}$-normal when each $R(P)$ is transitive.

A mapping $m$ of $A P A R$ into $2_{f}^{P A R}$ and of $A F O R$ into $2^{O B}$ is called assignment on $\mathcal{F}$. The pair $\mathcal{M}=(\mathcal{F}, m)$ is called model on $\mathcal{F} . \models_{\mathcal{M}} A$ - the truth in $\mathcal{M}$ of a formula $A$ - is defined in the following way :

- For every $A \in C F O R, \models_{\mathcal{M}} A$ iff $\tilde{m}(A)=O B$.

where $\tilde{m}$ is the mapping of $C P A R$ into $2_{f}^{P A R}$ and of $C F O R$ into $2^{O B}$ defined by induction in the following way :

- For every atomic parameter $\alpha, \tilde{m}(\alpha)=m(\alpha)$,

- For every ?, $\Delta \in C P A R, \tilde{m}(? \cap \Delta)=\tilde{m}($ ? $) \cup \tilde{m}(\Delta)$,

- For every atomic formula $A, \tilde{m}(A)=m(A)$,

- For every $A \in C F O R, \tilde{m}(-A)=O B \backslash \widetilde{m}(A)$,

- For every $A, B \in C F O R, \widetilde{m}(A \wedge B)=\widetilde{m}(A) \cap \widetilde{m}(B)$,

- For every ? $\in C P A R$ and for every $A \in C F O R, \tilde{m}([?] A)=$ $\{x \in O B$ : for every $y \in O B$, if $x R(\widetilde{m}(?)) y$ then $y \in \widetilde{m}(A)\}$.

Direct calculations would lead to the conclusion that :

Theorem 2 Let $\mathcal{F}=(P A R, O B, R)$ be a frame. Let $m$ be an assignment on $\mathcal{F}$. For every ?, $\Delta \in C P A R, R(\tilde{m}(? \cap \Delta)) \subseteq R(\tilde{m}(?)) \cap$ $R(\widetilde{m}(\Delta))$. If $\mathcal{F}$ is standard then, for every?, $\Delta \in C P A R, R(\widetilde{m}(? \cap$ $\Delta))=R(\tilde{m}(?)) \cap R(\tilde{m}(\Delta))$.

Moreover :

Theorem 3 Let $\mathcal{F}=(P A R, O B, R)$ be a frame. Let $m$ be an assignment on $\mathcal{F}$. For every ?, $\Delta \in C P A R$, if $\operatorname{set}(?) \subseteq \operatorname{set}(\Delta)$ then $\tilde{m}(?) \subseteq \tilde{m}(\Delta)$ and $R(\tilde{m}(\Delta)) \subseteq R(\tilde{m}(?))$.

$\models_{\mathcal{F}} A$ - the truth in $\mathcal{F}$ of a formula $A$ - is defined in the following way : 
- For every $A \in C F O R, \models_{\mathcal{F}} A$ iff, for every model $\mathcal{M}$ on $\mathcal{F}$, $\models_{\mathcal{M}} A$.

Let $\Omega$ be a nonempty set of frames. $F_{\Omega} A$ - the validity in $\Omega$ of a formula $A-$ is defined in the following way :

- For every $A \in C F O R, \models_{\Omega} A$ iff, for every $\mathcal{F} \in \Omega, \models_{\mathcal{F}} A$.

Let

- $K_{L 2}$ be the set of all frames,

- $K_{L 2}^{S}$ be the set of all standard frames,

- $K_{L 2}(m, n, j, k)$ be the set of all $\underset{j k}{m n}$-normal frames,

- $K_{L 2}^{S}(m, n, j, k)$ be the set of all standard $\underset{j k}{m n}$-normal frames,

- $S 5_{L 2}$ be the set of all frames with equivalence relations (each $R(P)$ is an equivalence relation),

- $S 5_{L 2}^{S}$ be the set of all standard frames with equivalence relations.

\subsection{Axiomatic presentation}

Let $\Omega$ be a class of frames. By the logic of $\Omega$ we mean the set of formulas of $C F O R$ that are valid in $\Omega$. For the sake of brevity we denote this logic by $\Omega$ as well. Together with the classical tautologies, all the instances of the following schemata are axioms of $K_{L 2}$ :

- $[?](A \rightarrow B) \rightarrow([?] A \rightarrow[?] B)$, for every ? $\in C P A R$,

- $[?] A \rightarrow[\Delta] A$, for every ?, $\Delta \in C P A R$ such that ? $\sqsubseteq \Delta$.

Together with the modus ponens, all the instances of the following schema are rules of $K_{L 2}$ :

- From $A$ infer [?] $A$, for every ? $\in C P A R$.

In a standard way we define the notions of proof and derivability in the logic $\Omega$.

\subsection{Completeness}

It is easy to see that the following soundness theorem holds for logic $K_{L 2}$ :

Theorem 4 For every $A \in C F O R$, if $\vdash_{K_{L 2}} A$ then $\models_{K_{L 2}} A$. 
Let $O B$ be the set of the maximal consistent sets of formulas. Let $R$ be the mapping of $2_{f}^{A P A R}$ into the set of the binary relations on $O B$ defined in the following way:

- For every $P \subseteq_{f} A P A R$ and for every $x, y \in O B, x R(P) y$ iff, for every $? \in C P A R$ and for every $A \in C F O R$, if $\operatorname{set}(?) \subseteq P$ and [?] $A \in x$ then $A \in y$.

Let it be proved that the structure of the form $\mathcal{F}=(A P A R, O B, R)$ is a frame:

$\mathcal{F}$ is a frame Let $P, Q \subseteq_{f} A P A R$, let $x, y \in O B$ be such that $x$ $R(P \cup Q) y$. Therefore, for every ? $\in C P A R$ and for every $A \in C F O R$, if $\operatorname{set}(?) \subseteq P \cup Q$ and [?] $A \in x$ then $A \in y$. Since $P \subseteq P \cup Q$ and $Q \subseteq P \cup Q$, then, for every $? \in C P A R$ and for every $A \in C F O R$, if set(?) $\subseteq P$ and [?] $A \in x$ then $\operatorname{set}(?) \subseteq P \cup Q$ and $A \in y$ and if $\operatorname{set}(?) \subseteq Q$ and [?] $A \in x$ then,

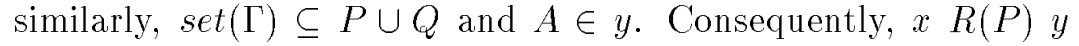
and $x R(Q) y$. Therefore, $R(P \cup Q) \subseteq R(P) \cap R(Q)$.

Let $m$ be an assignment on $\mathcal{F}$ defined in the following way :

- For every atomic parameter $\alpha, m(\alpha)=\{\alpha\}$,

- For every atomic formula $A, m(A)=\{x \in O B: A \in x\}$.

Let $\mathcal{M}=(\mathcal{F}, m)$. The proof is done by induction on ? that, for every $? \in C P A R, \tilde{m}(?)=\operatorname{set}(?):$

BASIS For every atomic parameter $\alpha, \tilde{m}(\alpha)=m(\alpha)=\{\alpha\}=\operatorname{set}(\alpha)$.

Hypothesis There is ?, $\Delta \in C P A R$ such that $\tilde{m}(?)=\operatorname{set}(?)$ and $\tilde{m}(\Delta)=\operatorname{set}(\Delta)$.

STEP $\tilde{m}(? \cap \Delta)=\tilde{m}(?) \cup \tilde{m}(\Delta)=$, by the hypothesis, set(?) $\cup$ $\operatorname{set}(\Delta)=\operatorname{set}(? \cap \Delta)$.

The proof is done by induction on $A$ that, for every $A \in C F O R$, $\tilde{m}(A)=\{x \in O B: A \in x\}$.

BAsIs For every atomic formula $A, \widetilde{m}(A)=m(A)=\{x \in O B$ : $A \in x\}$.

Hypothesis There is $A \in C F O R$ such that $\tilde{m}(A)=\{x \in O B$ : $A \in x\}$.

STEP For every ? $\in C P A R$, let $x \in O B$ be such that [?]A $\in x$. Consequently, for every $y \in O B$, if $x R(\widetilde{m}(?)) y$ then $A \in y$ 
and, by the hypothesis, $y \in \tilde{m}(A)$. Therefore, $x \in \tilde{m}([?] A)$.

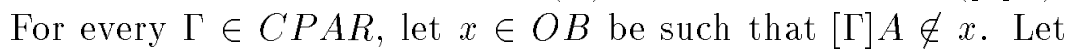
$y$ be a maximal consistent set of formulas containing $\{-A\} \cup$ $\{B \in C F O R:[?] B \in x\}$. Direct calculations would lead to the conclusion that $x R(\tilde{m}(?)) y$. Therefore, $x \notin \widetilde{m}([?] A)$.

Therefore :

Theorem 5 For every $A \in C F O R$, if $\models_{K_{L 2}}$ A then $\vdash_{K_{L 2}} A$.

In order to obtain the completeness result for $K_{L 2}$ with respect to its standard frames, we apply the method of copying originated in Vakarelov [26] in the context of the modal logics for knowledge representation systems.

\subsection{Copying}

Let $\mathcal{F}=(P A R, O B, R)$ and $\mathcal{F}^{\prime}=\left(P A R, O B^{\prime}, R^{\prime}\right)$ be frames. Let $I$ be a set of mappings of $O B$ into $O B^{\prime} . I$ is a copying of $\mathcal{F}$ into $\mathcal{F}^{\prime}$ whenever the following conditions are satisfied :

- For every $x^{\prime} \in O B^{\prime}$, there is $f \in I$ and there is $x \in O B$ such that $f(x)=x^{\prime}$,

- For every $f, g \in I$ and for every $x, y \in O B$, if $f(x)=g(y)$ then $x=y$,

- For every $P \subseteq_{f} P A R$, for every $f \in I$ and for every $x, y \in O B$, $x R(P) y$ iff there is $g \in I$ such that $f(x) R^{\prime}(P) g(y)$.

It is easy to show that :

Theorem 6 Let $\mathcal{F}=(P A R, O B, R)$ and $\mathcal{F}^{\prime}=\left(P A R, O B^{\prime}, R^{\prime}\right)$ be frames. Let $I$ be a copying of $\mathcal{F}$ into $\mathcal{F}^{\prime}$. Let $m$ be an assignment on $\mathcal{F}$. Let $m^{\prime}$ be the assignment on $\mathcal{F}^{\prime}$ defined in the following way:

- For every atomic parameter $\alpha, m^{\prime}(\alpha)=m(\alpha)$,

- For every atomic formula $A, m^{\prime}(A)=\{f(x): f \in I$ and $x \in$ $m(A)\}$.

Then for every $\alpha \in C P A R, m^{\prime}(\alpha)=m(\alpha)$ and for every $A \in C F O R$, $\widetilde{m^{\prime}}(A)=\{f(x): f \in I$ and $x \in \widetilde{m}(A)\}$. 


\subsection{Standard completeness}

Let $\mathcal{F}=(P A R, O B, R)$ be a frame. For every $P \subseteq_{f} P A R$, let $\sigma(P)$ be the mapping of $O B \times O B$ into $2^{O B}$ defined in the following way : for every $x, y \in O B, \sigma(P)(x, y)=\emptyset$ if $x R(P) y$, otherwise $\sigma(P)(x, y)=$ $O B$. Observe that the set $\mathcal{B}=2^{O B}$ can be treated as a Boolean ring where :

- $0_{\mathcal{B}}=\emptyset$,

- $1_{\mathcal{B}}=O B$,

- $A+{ }_{B} B=(A \backslash B) \cup(B \backslash A)$, consequently : $A+\mathcal{B} A=\emptyset$,

- $A \times_{\mathcal{B}} B=A \cap B$.

Therefore, for every $A, B \in 2^{O B}$, there exists exactly one $X \in 2^{O B}$ such that $A+_{\mathcal{B}} X=B$, namely : $X=(A \backslash B) \cup(B \backslash A)$. Let $I$ be the set of the mappings of $2_{f}^{P A R} \times P A R$ into $2^{O B}$. Let $O B^{\prime}=O B \times I$. For every $P \subseteq_{f} P A R$, let $R^{\prime}(P)$ be the binary relation on $O B^{\prime}$ defined in the following way :

- For every $f, g \in I$ and for every $x, y \in O B,(x, f) R^{\prime}(P)(y, g)$ iff :

- For every $O \subseteq_{f} P A R$ and for every $\alpha \in P A R$, if $\alpha \in O$ and $\alpha \in P$ then $g(O, \alpha)=f(O, \alpha)$ and

- For every $O \subseteq_{f} P A R, \Sigma_{\alpha \in O} f(O, \alpha)+g(O, \alpha)=\sigma(O)(x, y)$.

Lemma 1 Let $P \subseteq_{f} P A R$, let $f \in I$ and let $x, y \in O B$ be such that there is $g \in I$ such that $(x, f) R^{\prime}(P)(y, g)$. Then $x R(P) y$.

Proof : Let $P \subseteq_{f} P A R$, let $f \in I$ and let $x, y \in O B$ be such that there is $g \in I$ such that $(x, f) R^{\prime}(P)(y, g)$. Consequently, for every $O \subseteq_{f} P A R$ and for every $\alpha \in P A R$, if $\alpha \in O$ and $\alpha \in P$ then $g(O, \alpha)=f(O, \alpha)$ and, for every $O \subseteq_{f} P A R, \Sigma_{\alpha \in O} f(O, \alpha)+$ $g(O, \alpha)=\sigma(O)(x, y)$. Therefore, for every $\alpha \in P A R$, if $\alpha \in P$ then $g(P, \alpha)=f(P, \alpha)$. Consequently, $\sigma(P)(x, y)=\emptyset$. Therefore, $x R(P)$ $y$.

$\dashv$

Lemma 2 Let $P \subseteq_{f} P A R$, let $f \in I$ and let $x, y \in O B$ be such that $x R(P) y$. Then there is $g \in I$ such that $(x, f) R^{\prime}(P)(y, g)$. 
Proof : Let $P \subseteq_{f} P A R$, let $f \in I$ and let $x, y \in O B$ be such that $x R(P) y$. Let $\Phi$ be a mapping of $2_{f}^{P A R}$ into $P A R$ such that, for every $O \subseteq_{f} P A R$, if $O \nsubseteq P$ then $\Phi(O) \in O \backslash P$. We have to find a mapping $g \in I$ such that $(x, f) R^{\prime}(P)(y, g)$. Let $g$ be the mapping of $2_{f}^{P A R} \times P A R$ into $2^{O B}$ such that for every $O \subseteq_{f} P A R$ and for every $\alpha \in P A R$, the following conditions are satisfied :

- If $\alpha \in O$ and $\alpha \in P$ then $g(O, \alpha)=f(O, \alpha)$,

- If $\alpha \in O$ and $\alpha \notin P$ then either $\alpha=\Phi(O)$ in which case $g(O, \alpha)=$ $\Sigma_{\alpha \in O \backslash P} f(O, \alpha)+\sigma(O)(x, y)$ or $\alpha \neq \Phi(O)$ in which case $g(O, \alpha)=$ $\emptyset$,

- If $\alpha \notin O$ then $g(O, \alpha)=\emptyset$.

It is easy to verify that $(x, f) R^{\prime}(P)(y, g)$.

$\dashv$

Observe that, for every $f \in I, f$ can be identified with the mapping of $O B$ into $O B^{\prime}$ defined by : for every $x \in O B, f(x)=(x, f)$.

Lemma 3 The structure $\mathcal{F}^{\prime}=\left(P A R, O B^{\prime}, R^{\prime}\right)$ is a standard frame and $I$ - considered as a set of mappings of $O B$ into $O B^{\prime}-$ is a copying of $\mathcal{F}$ into $\mathcal{F}^{\prime}$.

Proof : The proof is done that $\mathcal{F}^{\prime}$ is a standard frame and $I$ is a copying of $\mathcal{F}$ into $\mathcal{F}^{\prime}$.

$\mathcal{F}^{\prime}$ is a standard frame - Let $P, Q \subseteq_{f} P A R$, let $f, g \in I$ and let $x, y \in O B$ be such that $(x, f) R^{\prime}(P \cup Q)(y, g)$. Consequently, for every $O \subseteq_{f} P A R$ and for every $\alpha \in P A R$, if $\alpha \in O$ and $\alpha \in P \cup Q$ then $g(O, \alpha)=f(O, \alpha)$ and, for every $O \subseteq_{f} P A R, \Sigma_{\alpha \in O} f(O, \alpha)+g(O, \alpha)=\sigma(O)(x, y)$. Since $P \subseteq P \cup Q$ and $Q \subseteq P \cup Q$, then, for every $O \subseteq_{f} P A R$ and for every $\alpha \in P A R$, if $\alpha \in O$ and $\alpha \in P$ then $\alpha \in P \cup Q$ and $g(O, \alpha)=f(O, \alpha)$ and if $\alpha \in O$ and $\alpha \in Q$ then, similarly, $\alpha \in P \cup Q$ and $g(O, \alpha)=f(O, \alpha)$. Therefore, $(x, f) R^{\prime}(P)(y, g)$ and $(x, f) R^{\prime}(Q)(y, g)$. Consequently, $R^{\prime}(P \cup Q) \subseteq R^{\prime}(P) \cup R^{\prime}(Q)$.

- Let $P, Q \subseteq_{f} P A R$, let $f, g \in I$ and let $x, y \in O B$ be such that $(x, f) R^{\prime}(P)(y, g)$ and $(x, f) R^{\prime}(Q)(y, g)$. Therefore, for every $O \subseteq_{f} P A R$ and for every $\alpha \in P A R$, if $\alpha \in O$ and $\alpha \in P$ then $g(O, \alpha)=f(O, \alpha)$ and if $\alpha \in O$ and $\alpha \in Q$ then $g(O, \alpha)=f(O, \alpha)$ and, for every $O \subseteq_{f} P A R$, 
$\Sigma_{\alpha \in O} f(O, \alpha)+g(O, \alpha)=\sigma(O)(x, y)$. Consequently, for every $O \subseteq_{f} P A R$ and for every $\alpha \in P A R$, if $\alpha \in O$ and $\alpha \in P \cup Q$ then $\alpha \in P$ and $g(O, \alpha)=f(O, \alpha)$ or $\alpha \in Q$ and $g(O, \alpha)=f(O, \alpha)$. Therefore, $(x, f) R^{\prime}(P \cup Q)(y, g)$. Consequently, $R^{\prime}(P \cup Q)=R^{\prime}(P) \cup R^{\prime}(Q)$.

$I$ is a copying of $\mathcal{F}$ into $\mathcal{F}^{\prime}$ This is a direct consequence of the lemmas 1 and 2.

$\dashv$

Consequently, it follows that the completeness theorem with respect to the class of standard frames holds :

Theorem 7 For every $A \in C F O R$, if $\models_{K_{L 2}^{S}} A$ then $\vdash_{K_{L 2 S}} A$.

\subsection{Extensions}

This subsection presents two extensions $-S 5_{L 2}$ and $K_{L 2}(m, n, j, k)$ - of $L 2$.

\subsection{1 $S 5_{L 2}$}

Together with the axioms and the rules of $K_{L 2}$, all the instances of the following schemata are axioms of $S 5_{L 2}$ :

- [?]A $\rightarrow A$, for every ? $\in C P A R$,

- $\langle ?\rangle[?] A \rightarrow[?] A$, for every $? \in C P A R$.

It is easy to see the soundness of logic $S 5_{L 2}$ :

Theorem 8 For every $A \in C F O R$, if $\vdash_{S 5_{L 2}}$ A then $\models_{S 5_{L 2}} A$.

Moreover, the structure of the form $\mathcal{F}=(A P A R, O B, R)$ defined in the subsection 2.4 is a frame with equivalence relations. Therefore we have :

Theorem 9 For every $A \in C F O R$, if $\models_{S 5_{L 2}} A$ then $\vdash_{S 5_{L 2}} A$.

Let $\mathcal{F}=(P A R, O B, R)$ be a frame with equivalence relations. For every $P \subseteq_{f} P A R$, let $\sigma(P)$ be the mapping of $O B \times O B$ into $2^{O B}$ defined in the following way : for every $x, y \in O B, \sigma(P)(x, y)=$ $R(P)(x)+R(P)(y)$. Let $I$ be the set of the mappings of $2_{f}^{P A R} \times P A R$ into $2^{O B}$. It can easily be proved that the argument of the subsection 2.6 applies : 
Lemma 4 The structure of the form $\mathcal{F}^{\prime}=\left(P A R, O B^{\prime}, R^{\prime}\right)$ defined in the subsection 2.6 is a standard frame with equivalence relations and $I$ is a copying of $\mathcal{F}$ into $\mathcal{F}^{\prime}$.

Consequently :

Theorem 10 For every $A \in C F O R$, if $\models_{S 5_{L 2}^{S}} A$ then $\vdash_{S 5_{L 2}} A$.

\subsection{2 $K_{L 2}(m, n, j, k)$}

Let $m, n, j, k \geq 0$. Together with the axioms and the rules of $K_{L 2}$, all the instances of the following schema are axioms of $K_{L 2}(m, n, j, k)$ :

- $\langle ?\rangle^{m}[?]^{n} A \rightarrow[?]^{j}\langle ?\rangle^{k} A$, for every $? \in C P A R$.

Theorem 11 For every $A \in C F O R$, if $\vdash_{K_{L 2}(m, n, j, k)}$ A then $\models_{K_{L 2}(m, n, j, k)}$ A.

The structure of the form $\mathcal{F}=(A P A R, O B, R)$ defined in the subsection 2.4 is an ${ }_{i k}^{m n}$-normal frame. Therefore we have the following completeness theorem for logics $K_{L 2}(m, n, j, k)$ :

Theorem 12 For every $A \in C F O R$, if $\models_{K_{L 2}(m, n, j, k)}$ A then $\vdash_{K_{L 2}(m, n, j, k)}$ A.

In what follows we present examples of logics of the form $K_{L 2}(m, n, j, k)$ for which the completeness theorem with respect to the standard frames holds. Let $\mathcal{F}=(P A R, O B, R)$ be an ${ }_{j k}^{m n}$-normal frame. For every $P \subseteq_{f} P A R$, let $\sigma(P)$ be the mapping of $O B \times O B$ into $2^{O B}$ defined in the following way: for every $x, y \in O B, \sigma(P)(x, y)=\emptyset$ if $x$ $R(P) y$, otherwise $\sigma(P)(x, y)=O B$. Let $I$ be the set of the mappings of $2_{f}^{P A R} \times P A R$ into $2^{O B}$. If $m=0, n=1, j=0$ and $k=0$, then the argument of the subsection 2.6 applies :

Lemma 5 If $m=0, n=1, j=0$ and $k=0$ then the structure of the form $\mathcal{F}^{\prime}=\left(P A R, O B^{\prime}, R^{\prime}\right)$ defined in the subsection 2.6 is a standard reflexive frame and $I$ is a copying of $\mathcal{F}$ into $\mathcal{F}^{\prime}$.

Consequently :

Theorem 13 For every $A \in C F O R$, if $\models_{K_{L 2}^{S}(0,1,0,0)} A$ then $\vdash_{K_{L 2}(0,1,0,0)}$ A. 
If $m=1, n=1, j=0$ and $k=0$ then the argument of the subsection 2.6 applies as well :

Lemma 6 If $m=1, n=1, j=0$ and $k=0$ then the structure of the form $\mathcal{F}^{\prime}=\left(P A R, O B^{\prime}, R^{\prime}\right)$ defined in the subsection 2.6 is a standard symmetric frame and $I$ is a copying of $\mathcal{F}$ into $\mathcal{F}^{\prime}$.

Consequently, we have the completeness theorem for logic $K_{L 2}(1,1,0,0)$ with respect to its standard frames:

Theorem 14 For every $A \in C F O R$, if $\models_{K_{L 2}^{S}(1,1,0,0)}$ A then $\vdash_{K_{L 2}(1,1,0,0)}$ A.

If $m+j \geq 2$ then the proof of the standard completeness of $K_{L 2}(m, 1, j, 0)$ and the proof of the standard completeness of $K_{L 2}(m, 0, j, 0)$ are open. If $n+k \geq 2$ then let $O B^{\prime}=O B \times\left(I^{O B \times I} \times I\right)$. For every $P \subseteq_{f} P A R$, let $R^{\prime}(P)$ be the binary relation on $O B^{\prime}$ defined in the following way:

- For every $f_{1}, g_{1} \in I^{O B \times I}$, for every $f_{2}, g_{2} \in I$ and for every $x, y \in O B,\left(x,\left(f_{1}, f_{2}\right)\right) R^{\prime}(P)\left(y,\left(g_{1}, g_{2}\right)\right)$ iff :

- For every $O \subseteq_{f} P A R$ and for every $\alpha \in P A R$, if $\alpha \in O$ and $\alpha \in P$ then $g_{1}\left(x, f_{2}\right)(O, \alpha)=f_{2}(O, \alpha)$,

- For every $O \subseteq_{f} P A R, \Sigma_{\alpha \in O} f_{2}(O, \alpha)+g_{1}\left(x, f_{2}\right)(O, \alpha)=$ $\sigma(O)(x, y)$.

The proofs of the three following lemmas are similar to the proofs of the lemmas 1,2 and 3 :

Lemma 7 Let $P \subseteq_{f} P A R$, let $f_{2} \in I$ and let $x, y \in O B$ be such that there is $G \in I$ such that for every $f_{1}, g_{1} \in I^{O B \times I}$ and for every $g_{2} \in I$, if $g_{1}\left(x, f_{2}\right)=G$ then $\left(x,\left(f_{1}, f_{2}\right)\right) R^{\prime}(P)\left(y,\left(g_{1}, g_{2}\right)\right)$. Then $x R(P) y$.

Lemma 8 Let $P \subseteq_{f} P A R$, let $f_{2} \in I$ and let $x, y \in O B$ be such that $x R(P) y$. There is $G \in I$ such that for every $f_{1}, g_{1} \in I^{O B \times I}$ and for every $g_{2} \in I$, if $g_{1}\left(x, f_{2}\right)=G$ then $\left(x,\left(f_{1}, f_{2}\right)\right) R^{\prime}(P)\left(y,\left(g_{1}, g_{2}\right)\right)$.

Lemma 9 The structure of the form $\mathcal{F}^{\prime}=\left(P A R, O B^{\prime}, R^{\prime}\right)$ is a standard ${ }_{j k}^{m n}$-normal frame and $I^{O B \times I} \times I-$ considered as a set of mappings of $O B$ into $O B^{\prime}-$ is a copying of $\mathcal{F}$ into $\mathcal{F}^{\prime}$.

Consequently :

Theorem 15 If $n+k \geq 2$, then for every $A \in C F O R$, if $\models_{K_{L 2}^{S}(m, n, j, k)}$ A then $\vdash_{K_{L 2}(m, n, j, k)} A$. 


\subsection{Examples}

Whereas several extensions of $L 2$ have a remote relationship with information systems, several extensions, on the other hand, have already been considered by many authors in the context of the modal logics for information systems.

$S 5_{L 2}^{S}$ is the class of standard frames in which each $R(m(?))$ is a relation of equivalence. In the context of the modal logics for information systems, it is the class of the frames of indiscernibility introduced by Orlowska [18].

One can easily extend the proof of the standard completeness of $K_{L 2}(1,1,0,0)$ - the class of the frames where each $R(m(?))$ is symmetric - to the class of the frames where each $R(m(?))$ is both reflexive and symmetric. This class is the class of the frames of strong similarity.

\section{Modal logics L3}

In this section we consider a class $L 3$ of relative modal logics where the parameters are defined by the operations $\cap$ and $\cup$ in such a way that, in the relational semantics of these logics, $R(? \cap \Delta)=R(?) \cap R(\Delta)$ and $R(? \cup \Delta)=R(?) \cup R(\Delta)$. We present axiomatization of various classes of these logics and we discuss their completeness.

\subsection{Language}

The language of any modal logic from class $L 3$ is obtained from the language of class $L 2$ by adjoining the operation of union to the set of operations acting on parameter expressions. Let $A P A R$ be a nonempty set of atomic parameters. The set $C P A R$ of the complex parameters is defined by induction in the following way :

- Every atomic parameter is a complex parameter,

- For every ?, $\Delta \in C P A R, ? \cap \Delta \in C P A R$,

- For every ?, $\Delta \in C P A R, ? \cup \Delta \in C P A R$.

Let set be the mapping of $C P A R$ into $2_{f}^{2 A P A R}$ - the set of the finite sets of finite subsets of $A P A R$ - defined by induction in the following way :

- For every atomic parameter $\alpha, \operatorname{set}(\alpha)=\{\{\alpha\}\}$, 
- For every ?, $\Delta \in C P A R, \operatorname{set}(? \cap \Delta)=\operatorname{set}(?) \cup \operatorname{set}(\Delta)$,

- For every ?, $\Delta \in C P A R, \operatorname{set}(? \cup \Delta)=\{S \cup T: S \in \operatorname{set}($ ? $)$ and $T \in \operatorname{set}(\Delta)\}$.

Direct calculations would lead to the conclusion that :

Theorem 16 For every $P \subseteq_{f} 2_{f}^{A P A R}$, there is $\in C P A R$ such that $\operatorname{set}(?)=P$.

Let $\sqsubseteq$ be the binary relation on $C P A R$ defined in the following way :

- For every ?, $\Delta \in C P A R$, ? $\sqsubseteq \Delta$ iff for every $S \in \operatorname{set}($ ?) there is $T \in \operatorname{set}(\Delta)$ such that $T \subseteq S$.

As remarked in section 2.1, if the expressions ? and $\Delta$ are considered as Boolean formulas of the classical propositional calculus, then one can easily prove that :

- ? $\sqsubseteq \Delta$ iff $\Delta \rightarrow$ ? is classically valid.

Let $A F O R$ be a nonempty set of atomic formulas. The set $C F O R$ of the complex formulas is defined by induction in the following way:

- Every atomic formula is a complex formula,

- For every $A \in C F O R, \neg A \in C F O R$,

- For every $A, B \in C F O R, A \wedge B \in C F O R$,

- For every ? $\in C P A R$ and for every $A \in C F O R$, [?] $A \in C F O R$.

For every ? $\in C P A R$ and for every $A \in C F O R$, let $\langle ?\rangle A=\neg[?] \neg A$.

\subsection{Semantical study}

A frame for $L 3$ is a relational structure of the form $\mathcal{F}=(P A R, O B, R)$ where:

- $P A R$ is a nonempty set of parameters,

- $O B$ is a nonempty set of objects,

- $R$ is a mapping of $2_{f}^{2_{f}^{P A R}}$ - the set of the finite sets of finite subsets of $P A R$ - into the set of the binary relations on $O B$ such that, for every $P, Q \subseteq_{f} 2_{f}^{P A R}, R(P \cup Q) \subseteq R(P) \cap R(Q)$ and $R(\{S \cup T: S \in P$ and $T \in Q\})=R(P) \cup R(Q)$. 
Throughout section 3, by "frame" we always mean a frame for $L 3 . \mathcal{F}$ is standard when, for every $P, Q \subseteq \subseteq_{f}^{P A R}, R(P \cup Q)=R(P) \cap R(Q)$. A mapping $m$ of $A P A R$ into $2_{f}^{2_{f}^{P A R}}$ and of $A F O R$ into $2^{O B}$ is called assignment on $\mathcal{F}$. The pair $\mathcal{M}=(\mathcal{F}, m)$ is called model on $\mathcal{F} . \models_{\mathcal{M}} A$ - the truth in $\mathcal{M}$ of a formula $A$ - is defined in the following way :

- For every $A \in C F O R, \models_{\mathcal{M}} A$ iff $\tilde{m}(A)=O B$.

where $\widetilde{m}$ is the mapping of $C P A R$ into $2_{f}^{2_{f}^{P A R}}$ and of $C F O R$ into $2^{O B}$ defined by induction in the following way :

- For every atomic parameter $\alpha, \tilde{m}(\alpha)=m(\alpha)$,

- For every ?, $\Delta \in C P A R, \tilde{m}(? \cap \Delta)=\tilde{m}($ ? $) \cup \tilde{m}(\Delta)$,

- For every ?, $\Delta \in C P A R, \tilde{m}(? \cup \Delta)=\{S \cup T: S \in \tilde{m}($ ? $)$ and $T \in \tilde{m}(\Delta)\}$,

- For every atomic formula $A, \tilde{m}(A)=m(A)$,

- For every $A \in C F O R, \tilde{m}(\neg A)=O B \backslash \tilde{m}(A)$,

- For every $A, B \in C F O R, \tilde{m}(A \wedge B)=\tilde{m}(A) \cap \tilde{m}(B)$,

- For every ? $\in C P A R$ and for every $A \in C F O R, \widetilde{m}([?] A)=$ $\{x \in O B$ : for every $y \in O B$, if $x R(\widetilde{m}(?)) y$ then $y \in \widetilde{m}(A)\}$.

It could easily be observed that :

- For every ?, $\Delta \in C P A R$, if ? $\sqsubseteq \Delta$ then $R(\widetilde{m}(\Delta)) \subseteq R(\widetilde{m}(?))$.

Moreover, direct calculations would lead to the conclusion that:

Theorem 17 Let $\mathcal{F}=(P A R, O B, R)$ be a frame. Let $m$ be an assignment on $\mathcal{F}$. For every ?, $\Delta \in C P A R, R(\widetilde{m}(? \cap \Delta)) \subseteq R(\tilde{m}(?)) \cap$ $R(\tilde{m}(\Delta))$ and $R(\tilde{m}(? \cup \Delta))=R(\tilde{m}(?)) \cup R(\tilde{m}(\Delta))$. If $\mathcal{F}$ is standard then, for every?, $\Delta \in C P A R, R(\tilde{m}(? \cap \Delta))=R(\tilde{m}(?)) \cap R(\tilde{m}(\Delta))$.

Moreover :

Theorem 18 Let $\mathcal{F}=(P A R, O B, R)$ be a frame. Let $m$ be an assignment on $\mathcal{F}$. For every?, $\Delta, \Lambda \in C P A R$, if, for every $S \in \operatorname{set}($ ? $\cup \Delta)$, there is $T \in \operatorname{set}(\Lambda)$ such that $T \subseteq S$ then, for every $S \in \widetilde{m}(? \cup \Delta)$, there is $T \in \tilde{m}(\Lambda)$ such that $T \subseteq S$ and $R(\widetilde{m}(\Lambda)) \subseteq R(\tilde{m}(?)) \cup$ $R(\tilde{m}(\Delta))$.

The notions of truth of a formula in a frame, validity of a formula in a class of frames and logic of a class of frames are defined in a usual way. We distinguish the following classes of frames : 
- $K_{L 3}$ is the set of all frames,

- $K_{L 3}^{S}$ is the set of all standard frames,

- $K_{L 3}(m, n, j, k)$ is the set of all $\underset{j k}{m n}$-normal frames,

- $K_{L 3}^{S}(m, n, j, k)$ is the set of all standard ${ }_{j k}^{m n}$-normal frames,

- $S 5_{L 3}^{S}$ is the set of all standard frames with equivalence relations.

Observe that :

Theorem 19 Let $\mathcal{F}=(P A R, O B, R)$ be a frame with equivalence relations. If $\mathcal{F}$ is standard then all the instances of the following schema are true in $\mathcal{F}$ :

- [?]A $\rightarrow[\Delta] A \vee[\Lambda] A$, for every ?, $\Delta, \Lambda \in C P A R$ such that? $\sqsubseteq$ $\Delta \cap \Lambda$.

Proof Suppose that $\mathcal{F}$ is standard and let $m$ be an assignment on $\mathcal{F}$. Let ?, $\triangle, \Lambda \in C P A R$ be such that : ? $\square \cap \Lambda$ and let $A \in C F O R$. Let $x \in O B$. If $x \notin \widetilde{m}([\Delta] A \vee[\Lambda] A)$ then there exists $y \in O B$ such that $x R(\tilde{m}(\Delta)) y$ and $y \notin \tilde{m}(A)$ and there exists $z \in O B$ such that $x$ $R(\tilde{m}(\Lambda)) z$ and $z \notin \tilde{m}(A)$. Then, $x R(\tilde{m}(\Delta \cup \Lambda)) y$ and $x R(\tilde{m}(\Delta \cup \Lambda))$ $z$. Since $R(\tilde{m}(\Delta \cup \Lambda))$ is a relation of equivalence, then $y R(\tilde{m}(\Delta \cup \Lambda))$ $z$ and either $y R(\tilde{m}(\Delta)) z$ or $y R(\tilde{m}(\Lambda)) z$. If $y R(\tilde{m}(\Delta)) z$ then $x$ $R(\widetilde{m}(\Delta)) z$ and $x R(\widetilde{m}(\Lambda)) z$. Since $\mathcal{F}$ is standard, then $x R(\widetilde{m}(\Delta \cap \Lambda))$ $z$. Since ? $\sqsubseteq \Delta \cap \Lambda$, then $x R(\tilde{m}(?)) z$ and $x \notin \tilde{m}([?] A)$. Similarly, if $y$ $R(\tilde{m}(\Lambda)) z$ then $x R(\tilde{m}(\Delta)) y$ and $x R(\tilde{m}(\Lambda)) y$. Since $\mathcal{F}$ is standard, then $x R(\tilde{m}(\Delta \cap \Lambda)) y$. Since ? $\sqsubseteq \Delta \cap \Lambda$, then $x R(\tilde{m}(?)) y$ and $x \notin \tilde{m}([?] A)$.

\subsection{Axiomatic presentation}

Together with the classical tautologies, all the instances of the following schemata are axioms of $K_{L 3}$ :

- [?] $(A \rightarrow B) \rightarrow([?] A \rightarrow[?] B)$, for every ? $\in C P A R$,

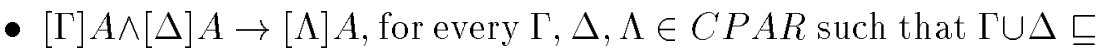
$\Lambda$.

Together with the modus ponens, all the instances of the following schema are rules of $K_{L 3}$ : 
- From $A$ infer [?] $A$, for every ? $\in C P A R$.

It can be easily observed that, for every ?, $\triangle \in C P A R$, all the instances of the following schemata are theorems of $K_{L 3}:[?] A \wedge[\Delta] A \leftrightarrow$ $[? \cup \Delta] A$ and $[?] A \vee[\Delta] A \rightarrow[? \cap \Delta] A$. Moreover, if $? \sqsubseteq \Delta$ then all the instances of the following schema are theorems of $K_{L 3}:[?] A \rightarrow[\Delta] A$.

\subsection{Completeness}

It is easy to see that the following soundness theorem holds for logic $K_{L 3}$ :

Theorem 20 For every $A \in C F O R$, if $\vdash_{K_{L 3}} A$ then $\models_{K_{L 3}} A$.

Let $O B$ be the set of the maximal consistent sets of formulas. Let $R$ be the mapping of $2_{f}^{2_{f}^{A P A R}}$ into the set of the binary relations on $O B$ defined in the following way :

- For every $P \subseteq_{f} 2_{f}^{A P A R}$ and for every $x, y \in O B, x R(P) y$ iff for every ? $\in C P A R$ and for every $A \in C F O R$, if for every $S \in \operatorname{set}(?)$ there is $T \in P$ such that $T \subseteq S$, then if [?]A $\in x$ then $A \in y$.

Let it be proved that the structure of the form $\mathcal{F}=(A P A R, O B, R)$ is a frame:

$\mathcal{F}$ is a frame Let $P, Q \subseteq_{f} 2_{f}^{A P A R}$, let $x, y \in O B$ be such that $x$ $R(P \cup Q) y$ and let it be proved that $x R(P) y$ and $x R(Q) y$. Therefore, for every ? $\in C P A R$ and for every $A \in C F O R$, if, for every $S \in \operatorname{set}(?)$, there is $T \in P \cup Q$ such that $T \subseteq S$ and [?] $A \in x$ then $A \in y$. Since $P \subseteq P \cup Q$ and $Q \subseteq P \cup Q$, then, for every ? $\in C P A R$ and for every $A \in C F O R$, if, for every $S \in \operatorname{set}(?)$, there is $T \in P$ such that $T \subseteq S$ and [?] $A \in x$ then, for every $S \in \operatorname{set}(?)$, there is $T \in P \cup Q$ such that $T \subseteq S$ and $A \in y$ and if, for every $S \in \operatorname{set}(?)$, there is $T \in Q$ such that $T \subseteq S$ and [?] $A \in x$ then, similarly, for every $S \in \operatorname{set}(?)$, there is $T \in P \cup Q$ such that $T \subseteq S$ and $A \in y$. Consequently, $x R(P)$ $y$ and $x R(Q) y$. Let $P, Q \subseteq_{f} 2_{f}^{A P A R}$, let $x, y \in O B$ be such that $x R(\{S \cup T: S \in P$ and $T \in Q\}) y$ and let it be proved that $x R(P) y$ or $x R(Q) y$. Assume that $x \overline{R(P)} y$ and $x \overline{R(Q)} y$. Then there exists? $\in C P A R$ and there exists $A \in C F O R$ such that : 
- for every $U \in \operatorname{set}($ ?) there is $S \in P$ such that $S \subseteq U$,

- [?] $A \in x$ and

- $A \notin y$,

and there exists $\Delta \in C P A R$ and there exists $B \in C F O R$ such that :

- for every $V \in \operatorname{set}(\Delta)$ there is $T \in Q$ such that $T \subseteq V$,

- $[\Delta] B \in x$ and

- $B \notin y$

Therefore, [?] $(A \vee B) \in x,[\Delta](A \vee B) \in x$ and $[? \cup \Delta](A \vee B) \in x$. Moreover, for every $W \in \operatorname{set}(? \cup \Delta)$, there is $U \in \operatorname{set}(?)$ and there is $V \in \operatorname{set}(\Delta)$ such that $W=U \cup V$ and, furthermore, there is $S \in P$ and there is $T \in Q$ such that $S \cup T \subseteq U \cup V$. Since $x R(\{S \cup T: S \in P$ and $T \in Q\}) y$ and $[? \cup \Delta](A \vee B) \in x$, then $A \vee B \in y$, a contradiction. Therefore, either $x R(P) y$ or $x R(Q) y$. Reciprocally, let $P, Q \subseteq_{f} 2_{f}^{A P A R}$, let $x, y \in O B$ be such that either $x R(P) y$ or $x R(Q) y$ and let it be proved that $x R(\{S \cup T: S \in P$ and $T \in Q\}) y$. Suppose that $x R(P)$ $y$. Then for every ? $\in C P A R$ and for every $A \in C F O R$, if for every $S \in \operatorname{set}($ ?) there is $T \in P$ such that $T \subseteq S$, then if [?] $A \in x$ then $A \in y$. Now, let $? \in C P A R$ be such that, for every $S \in \operatorname{set}(?)$ there is $T \cup U \in\{T \cup U: T \in P$ and $U \in Q\}$ such that $T \cup U \subseteq S$. Therefore, for every $S \in \operatorname{set}($ ?) there is $T \in P$ such that $T \subseteq S$. Now, for every $A \in C F O R$, if [?] $A \in x$ then $A \in y$. Then $x R(\{S \cup T: S \in P$ and $T \in Q\}) y$.

Let $m$ be the assignment on $\mathcal{F}$ defined in the following way :

- For every atomic parameter $\alpha, m(\alpha)=\{\{\alpha\}\}$,

- For every atomic formula $A, m(A)=\{x \in O B: A \in x\}$.

Let $\mathcal{M}=(\mathcal{F}, m)$. The proof is done by induction on ? that, for every $? \in C P A R, \tilde{m}(?)=\operatorname{set}(?)$.

BASIS For every atomic parameter $\alpha, \tilde{m}(\alpha)=m(\alpha)=\{\{\alpha\}\}=$ set (?).

Hypothesis There is ?, $\Delta \in C P A R$ such that $\tilde{m}(?)=\operatorname{set}(?)$ and $\tilde{m}(\Delta)=\operatorname{set}(\Delta)$.

STEP $\tilde{m}(? \cap \Delta)=\tilde{m}(?) \cup \tilde{m}(\Delta)=$, by the hypothesis, $\operatorname{set}(?) \cup$ $\operatorname{set}(\Delta)=\operatorname{set}(? \cap \Delta) . \tilde{m}(? \cup \Delta)=\{S \cup T: S \in \tilde{m}(?)$ and 
$T \in \tilde{m}(\Delta)\}=$, by the hypothesis, $\{S \cup T: S \in \operatorname{set}($ ? $)$ and $T \in \operatorname{set}(\Delta)\}=\operatorname{set}(? \cup \Delta)$.

The proof is done by induction on $A$ that, for every $A \in C F O R$, $\widetilde{m}(A)=\{x \in O B: A \in x\}$.

BASIS For every atomic formula $A, \tilde{m}(A)=m(A)=\{x \in O B$ : $A \in x\}$.

Hypothesis There is $A \in C F O R$ such that $\tilde{m}(A)=\{x \in O B$ : $A \in x\}$.

STEP For every $? \in C P A R$, let $x \in O B$ be such that [?]A $\in x$. Consequently, for every $y \in O B$, if $x R(\widetilde{m}(?)) y$ then $A \in y$ and, by the hypothesis, $y \in \widetilde{m}(A)$. Therefore, $x \in \tilde{m}([?] A)$. For every $? \in C P A R$, let $x \in O B$ be such that [?]A $\notin x$. Let $y$ be a maximal consistent set of formulas containing $\{-A\} \cup$ $\{B \in C F O R:[?] B \in x\}$. Direct calculations would lead to the conclusion that $x R(\widetilde{m}(?)) y$. Therefore, $x \notin \widetilde{m}([?] A)$.

Therefore :

Theorem 21 For every $A \in C F O R$, if $\models_{K_{L 3}} A$ then $\vdash_{K_{L 3}} A$.

\subsection{Copying}

Let $\mathcal{F}=(P A R, O B, R)$ and $\mathcal{F}^{\prime}=\left(P A R, O B^{\prime}, R^{\prime}\right)$ be frames. Let $I$ be a set of mappings of $O B$ into $O B^{\prime} . I$ is a copying of $\mathcal{F}$ into $\mathcal{F}^{\prime}$ whenever the following conditions are satisfied :

- For every $x^{\prime} \in O B^{\prime}$, there is $f \in I$ and there is $x \in O B$ such that $f(x)=x^{\prime}$,

- For every $f, g \in I$ and for every $x, y \in O B$, if $f(x)=g(y)$ then $x=y$,

- For every $P \subseteq_{f} 2_{f}^{P A R}$, for every $f \in I$ and for every $x, y \in O B$, $x R(P) y$ iff there is $g \in I$ such that $f(x) R^{\prime}(P) g(y)$.

Direct calculations would lead to the conclusion that :

Theorem 22 Let $\mathcal{F}=(P A R, O B, R)$ and $\mathcal{F}^{\prime}=\left(P A R, O B^{\prime}, R^{\prime}\right)$ be frames. Let $I$ be a copying of $\mathcal{F}$ into $\mathcal{F}^{\prime}$. Let $m$ be an assignment on $\mathcal{F}$. Let $m^{\prime}$ be the assignment on $\mathcal{F}^{\prime}$ defined in the following way:

- For every atomic parameter $\alpha, m^{\prime}(\alpha)=m(\alpha)$, 
- For every atomic formula $A, m^{\prime}(A)=\{f(x): f \in I$ and $x \in$ $m(A)\}$.

Then for every $A \in C F O R, \widetilde{m^{\prime}}(A)=\{f(x): f \in I$ and $x \in \widetilde{m}(A)\}$.

\subsection{Standard completeness}

Let $\mathcal{F}=(P A R, O B, R)$ be a frame. For every $P \subseteq_{f} 2_{o}^{P A R}$, let $\sigma(P)$ be the mapping of $O B \times O B$ into $2^{O B}$ such that, for every $x, y \in O B$, $\sigma(P)(x, y)=\emptyset$ if $x R(P) y$, otherwise $\sigma(P)(x, y)=O B$. Let $I$ be the set of the mappings of $2_{f}^{2_{o}^{P A R}} \times 2_{o}^{P A R}-2_{o}^{P A R}$ is the set of the singletons of $P A R$ and $2_{f}^{2_{o}^{P A R}}$ is the set of the finite sets of singletons of $P A R$ - into $2^{O B}$. Let $O B^{\prime}=O B \times I$. For every $P \subseteq_{f} 2_{o}^{P A R}$, let $R^{\prime}(P)$ be the binary relation on $O B^{\prime}$ defined in the following way:

- For every $f, g \in I$ and for every $x, y \in O B,(x, f) R^{\prime}(P)(y, g)$ iff :

- For every $O \subseteq_{f} 2_{o}^{P A R}$ and for every $\alpha \in 2_{o}^{P A R}$, if $\alpha \in O$ and $\alpha \in P$ then $g(O, \alpha)=f(O, \alpha)$, and

- For every $O \subseteq_{f} 2_{o}^{P A R}, \Sigma_{\alpha \in O} f(O, \alpha)+g(O, \alpha)=\sigma(O)(x, y)$.

For every $P \subseteq_{f} 2_{f}^{P A R}$, let $R^{\prime}(P)$ be the binary relation on $O B^{\prime}$ defined in the following way:

- For every $f, g \in I$ and for every $x, y \in O B,(x, f) R^{\prime}(P)(y, g)$ iff there is a mapping $\phi$ of $P$ into $2_{0}^{P A R}$ such that:

- For every $S \in P, \phi(S) \subseteq S$,

- $(x, f) R^{\prime}(\{\phi(S): S \in P\})(y, g)$.

The proofs of the three following lemmas are similar to the proofs of the lemmas 1, 2 and 3 :

Lemma 10 Let $P \subseteq_{f} 2_{f}^{P A R}$, let $f, g \in I$ and let $x, y \in O B$ be such that $(x, f) R^{\prime}(P)(y, g)$. Then $x R(P) y$.

Lemma 11 Let $P \subseteq_{f} 2_{f}^{P A R}$, let $f \in I$ and let $x, y \in O B$ be such that $x R(P) y$. There is $g \in I$ such that $(x, f) R^{\prime}(P)(y, g)$.

Lemma 12 The structure of the form $\mathcal{F}^{\prime}=\left(P A R, O B^{\prime}, R^{\prime}\right)$ is a standard frame and $I$ - considered as a set of mappings of $O B$ into $O B^{\prime}$ - is a copying of $\mathcal{F}$ into $\mathcal{F}^{\prime}$. 
It follows that we have the following completeness theorem with respect to the standard frames:

Theorem 23 For every $A \in C F O R$, if $\models_{K_{L 3}^{S}}$ A then $\vdash_{K_{L 3}} A$.

\subsection{Extensions}

This subsection presents two extensions $-S 5_{L 3}$ and $K_{L 3}(m, n, j, k)-$ of the modal logic with strong and pseudo-weak accessibility relations.

\subsection{1 $S 5_{L 3}$}

Together with the axioms and the rules of $K_{L 3}$, all the instances of the following schemata are axioms of $S 5_{L 3}$ :

- [?]A $\rightarrow A$, for every ? $\in C P A R$,

- $\langle$ ? $\rangle A \rightarrow[?]\langle$ ? $\rangle A$, for every ? $\in C P A R$,

- [?]A $\rightarrow[\Delta] A \vee[\Lambda] A$, for every ?, $\Delta, \Lambda \in C P A R$ such that ? $\sqsubseteq$ $\Delta \cap \Lambda$.

Theorem 24 For every $A \in C F O R$, if $\vdash_{S 5_{L 3}} A$ then $\models_{S 5_{L 3}^{S}} A$.

Moreover :

Lemma 13 The structure of the form $\mathcal{F}=(A P A R, O B, R)$ defined in the subsection 3.4 is a standard frame with equivalence relations.

Proof : Suppose that $\mathcal{F}$ is not standard. Then there exists $P, Q \subseteq f$ $2_{f}^{A P A R}$ and there exists $x, y \in O B$ such that $x R(P) y, x R(Q) y$ and $x \overline{R(P \cup Q)} y$. Let $\Delta, \Lambda \in C P A R$ be such that $\operatorname{set}(\Delta)=P$ and $\operatorname{set}(\Lambda)$ $=Q$. Since $x \overline{R(P \cup Q)} y$, then there exists ? $\in C P A R$ and there exists $A \in C F O R$ such that :

- For every $S \in \operatorname{set}(?)$, there exists $T \in P \cup Q$ such that $T \subseteq S$.

- $[?] A \in x$.

- $A \notin y$.

Since $P \cup Q=\operatorname{set}(\Delta \cap \Lambda)$, then ? $\sqsubseteq \Delta \cap \Lambda$ and either $[\Delta] A \in x$ or $[\Lambda] A \in x$. Consequently, $A \in y:$ a contradiction.

$\dashv$

Therefore :

Theorem 25 For every $A \in C F O R$, if $\models_{S 5_{L 3}^{S}} A$ then $\vdash_{S 5_{L 3}} A$. 


\subsection{2 $K_{L 3}(m, n, j, k)$}

Let $m, n, j, k \geq 0$. Together with the axioms and the rules of $K_{L 3}$, all the instances of the following schema are axioms of $K_{L 3}(m, n, j, k)$ :

- $\langle ?\rangle^{m}[?]^{n} A \rightarrow[?]^{j}\langle ?\rangle^{k} A$, for every $? \in C P A R$.

We have the following soundness theorem :

Theorem 26 For every $A \in C F O R$, if $\vdash_{K_{L 3}(m, n, j, k)}$ A then $\models_{K_{L 3}(m, n, j, k)}$ A.

Observe that the structure of the form $\mathcal{F}=(A P A R, O B, R)$ defined in the subsection 3.4 is an $\underset{j k}{m n}$-normal frame. Therefore :

Theorem 27 For every $A \in C F O R$, if $\models_{K_{L 3}(m, n, j, k)}$ A then $\vdash_{K_{L 3}(m, n, j, k)}$ A.

The results analogous to theorems $13,14,15$ can be obtained for the logics of the class $L 3$. The proofs of the three following theorems are similar to the proofs of the theorems 13,14 and 15 :

Theorem 28 For every $A \in C F O R$, if $\models_{K_{L 3}^{S}(0,1,0,0)}$ A then $\vdash_{K_{L 3}(0,1,0,0)}$ A.

Theorem 29 For every $A \in C F O R$, if $\models_{K_{L 3}^{S}(1,1,0,0)}$ A then $\vdash_{K_{L 3}(1,1,0,0)}$ A.

Theorem 30 If $n+k \geq 2$ then, for every $A \in C F O R$, if $\models_{K_{L 3}^{S}(m, n, j, k)}$ A then $\vdash_{K_{L 3}(m, n, j, k)} A$.

\subsection{Examples}

The language of the relative modal logics of the class $L 3$ is defined by the operations $\cap$ and $U$. Therefore, it is a sublanguage of the language of the Boolean modal logic introduced by Gargov, Passy, Tinchev [11] [12] and $K_{L 3}$ is nothing but a fragment of $B M L$. As well, the extensions $K_{L 3}(m, n, j, k)$ are axiomatizable extensions of this fragment. In other respects, the relative modal logic $S 5_{L 3}$ is exactly the data analysis logic with local agreement $D A L L A$ introduced by Gargov [10] and further developed by Demri [5]. 


\section{Conclusion}

In this paper, we developed a general framework for presenting and studying modal logics based on frames with relative accessibility relations. We defined two major hierarchies of the classes of these logics: logics of class $L 2$ and logics of class $L 3$. For each class of logics we defined semantic structures of the two kinds: general structures and standard structures. We presented an axiomatization of several classes of logics from the given hierarchies and we studied their completeness with respect to both general and standard semantic structures. We showed that several modal logics for information systems are members of the classes $L 2$ and $L 3$.

For the modal logics of class $L 2$, the accessibility relations $R$ of the models $(P A R, O B, R, m)$ are parametrized by the elements of $2_{f}^{P A R}$, the set of the finite subsets of $P A R$. For the modal logics of class $L 3$, the accessibility relations $R$ are parametrized by the elements of

$2_{f}^{2_{f}^{P A R}}$, the set of the finite subsets of the set of the finite subsets of $P A R$. What would be the modal logic the models of which are relational structures of the form $(P A R, O B, R, m)$ where the accessibility relations $R$ are parametrized by the elements of the set of the finite subsets of the set of the finite subsets of the set of the finite subsets of $P A R$ ?

\section{Acknowledgement}

Special acknowledgement is heartly granted to Stéphane Demri who made several helpful comments for improving the readability of the paper.

\section{References}

[1] D. Arhangelsky and M. Taitslin. A logic for data description. A. Meyer and M. Taitslin (editors), Logic at Botik'89. Lecture Notes in Computer Science 363, 2-11, Springer-Verlag, 1989.

[2] P. Balbiani. A modal logic for data analysis. W. Penczek, A. Szalas (editors), Mathematical Foundations of Computer Science 
1996. Lecture Notes in Computer Science 1113, Springer-Verlag, $167-179,1996$.

[3] P. Balbiani. Axiomatization of logics based on Kripke models with relative accessibility relations. E. Orlowska (editor), Incomplete Information : Rough Set Analysis. Studies in Fuzziness and Soft Computing 13, 553-578, Physica Verlag, 1998.

[4] R. Danecki. Nondeterministic propositional dynamic logic with intersection is decidable. A. Skowron (editor), Computation Theory. Lecture Notes in Computer Science 208, 34-53, SpringerVerlag, 1985.

[5] S. Demri. The validity problem for DALLA is decidable. Bulletin of the Polish Academy of Sciences, Mathematics, Volume 4, $79-86,1996$.

[6] S. Demri. A completeness proof for a logic with an alternative necessity operator. Studia Logica, Volume 58, 99-112, 1997.

[7] S. Demri and E. Orłowska. Logical analysis of indiscernibility. E. Orłowska (editor), Incomplete Information : Rough Set Analysis. Studies in Fuzziness and Soft Computing 13, 347-380, Physica Verlag, 1998.

[8] R. Fagin, J. Halpern and M. Vardi. What can machines know? On the properties of knowledge in distributed systems. Journal of the ACM, Volume 39, 328-376, 1992.

[9] L. Fariñas del Cerro and E. Orłowska. $D A L-$ a logic for data analysis. Theoretical Computer Science, Volume 36, 251264, 1985.

[10] G. Gargov. Two completeness theorems in the logic for data analysis. Tech. Report 581, Institute of Computer Science, Polish Academy of Sciences, Warsaw, 1986.

[11] G. Gargov and S. Passy. A note on Boolean modal logic. P. Petkov (editor), Mathematical Logic. 299-309, Plenum Press, 1990.

[12] G. Gargov, S. Passy and T. Tinchev. Modal environment for Boolean speculations. D. Skordev (editor), Mathematical Logic and its Applications. 253-263, Plenum Press, 1987. 
[13] D. Harel. Dynamic logic. D. Gabbay and F. Guenthner (editors), Handbook of Philosophical Logic, Volume 2, Extensions of Classical Logic. 497-604, Reidel, 1984.

[14] W. van der Hoek and J.-J. Meyer. Making some issues of implicit knowledge explicit. Foundations of Computer Science, Volume 3, 193-223, 1992.

[15] B. Konikowska. A formal language for reasoning about indiscernibility. Bulletin of the Polish Academy of Sciences, Mathematics, Volume 35, 239-249, 1987.

[16] B. Konikowska. A logic for reasoning about relative similarity. Studia Logica, Volume 58, 185-226, 1997.

[17] E. Orłowska. Semantics of vague concepts. G. Dorn, P. Weingartner (editors), Foundations of Logic and Linguistics. Problems and Solutions. Selected Contributions to the 7th International Congress of Logic, Methodology and Philosophy of Science, Salzburg 1983. 465-482, Plenum Press, 1983.

[18] E. Orłowska. Logic of indiscernibility relations. A. Skow ron (editor), Computation Theory. Lecture Notes in Computer Science 208, 177-186, Springer-Verlag, 1985.

[19] E. Orłowska. Logic of nondeterministic information. Studia Logica, Volume 44, 93-102, 1985.

[20] E. Orłowska. Kripke models with relative accessibility and their application to inferences from incomplete information. G. Mirkowska, H. Rasiowa (editors), Mathematical Problems in Computation Theory. Banach Center Publications 21, 329-339, 1988 .

[21] E. Orłowska. Logic for reasoning about knowledge. Zeitschrift für Mathematische Logik und Grundlagen der Mathematik, Volume 35, 559-572, 1989.

[22] E. Orłowska. Studying incompleteness of information : a class of information logics. K. Kijania-Placek, J. Wolenski (editors), The Lvov Warsaw School and the Contemporary Philosophy. Kluwer, Dordrecht, 1997, 303-320.

[23] E. Orłowska (editor). Incomplete Information : Rough Set Analysis. Studies in Fuzziness and Soft Computing 13, PhysicaVerlag, 1998. 
[24] S. Read. Thinking about Logic. Oxford University Press, 1994.

[25] D. Vakarelov. Abstract characterization of some knowledge representation systems and the logic NIL of nondeterministic information. P. Jorrand, V. Sgurev (editors), Artificial Intelligence II, Methodology, Systems, Applications. North-Holland, 255-260, 1987.

[26] D. Vakarelov. Modal logics for knowledge representation systems. Theoretical Computer Science, Volume 90, 433-456, 1991.

[27] D. Vakarelov. A modal logic for similarity relations in Pawlak knowledge representation systems. Fundamenta Informaticæ, Volume 15, 61-79, 1991. 